\title{
Optogenetics takes more control
}

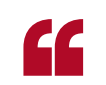

Two new studies now expand the optogenetic repertoire

The ability to control neuronal activity by light has already transformed neuroscience. Two new studies now expand the optogenetic repertoire by showing light-induced inhibition of synaptic release and control of gene transcription and chromatin modifications.

Reporting in Neuron, Tsien and colleagues applied the existing method of chromophore-assisted light inactivation (CALI) of proteins to presynaptic vesicular release proteins, with the aim of blocking vesicle release. They genetically fused miniSOG, an engineered flavoprotein, to the cytosolic termini of the SNARE proteins synaptophysin (SYP1) and synaptobrevin 2 (SYB2; from here on referred to as VAMP2). When miniSOG is activated by light of the appropriate wavelength $(480 \mathrm{~nm})$, it produces singlet oxygen, and this inactivates the nearby protein - that is, the protein fused to miniSOG, which in this case is SYP1 or VAMP2.

Illuminating cultured hippocampal neurons expressing miniSOG-VAMP2 or SYP1-miniSOG reduced the excitatory postsynaptic current (EPSC) amplitude by $30 \%$ and $83 \%$, respectively, indicating substantially reduced synaptic release. A FM464-dye imaging assay revealed that CALI had high spatial specificity, affecting only presynaptic terminals within an illuminated area.
The authors termed this method Inhibition of Synapses with CALI (InSynC) and tested it in organotypic slices and in vivo. They expressed SYP1-miniSOG in CA3 neurons in hippocampal slices. Illumination of a dendritic CA3-CA1 synapse depressed excitatory postsynaptic potentials (EPSPs) by $87 \%$ and reduced evoked EPSC amplitudes by $82 \%$. Furthermore, when Caenorhabditis elegans pan-neuronally expressing miniSOG-VAMP2 were illuminated for 5 minutes, their movement decreased on average by $\sim 80 \%$, with some worms showing complete paralysis. In accordance with this finding, illumination eliminated or reduced the number and amplitude of muscle EPSCs, indicating a reduction of synaptic inputs. Interestingly, the worms' movement had recovered $24 \mathrm{~h}$ later.

In a study in Nature, Zhang and colleagues describe a light-inducible transcriptional effector (LITE) system to control gene transcription. This system comprises a DNA-binding domain (based on transcription-activator-like effectors (TALEs)); the light-sensitive protein cryptochrome 2 (CRY2); the CRY2 interaction partner CIB1; and an effector domain. The TALE can be adapted to bind to the promoter region of any target gene and is fused to either CRY2 or CIB1; the effector is fused to the remaining component. A crucial aspect of LITE systems is that illumination with blue light causes CRY2 to bind CIB1 and that, as a result, the effector is recruited to the target promoter to induce or suppress (depending on the effector) transcription of the target gene.
The authors tested several LITE designs to target the gene neurogenin 2 (Neurog2) and found that, when transfected into cultured neurons, a system containing a truncated (but still light-sensitive) form of CRY2 (CRY2PHR) and the effector VP64 caused the most effective lightinduced Neurog 2 transcription. They developed a method for viral-vector delivery of a similar LITE system targeting Grm2 (which encodes metabotropic glutamate receptor 2 (mGluR2)) and applied it in vitro and in vivo. In primary cortical neurons, $4 \mathrm{~h}$ and $24 \mathrm{~h}$ of illumination increased Grm2 mRNA levels 4-fold and 7-fold, respectively. Importantly, mGluR2 protein levels increased accordingly. In the mouse prefrontal cortex in vivo, $12 \mathrm{~h}$ of illumination through a fiber-optic cannula caused a 2.5-fold increase in Grm 2 mRNA levels.

The authors also designed a LITE system in which CRY2PHR was fused to a histone effector domain, with the aim of changing the epigenetic state around a target gene rather than gene transcription directly. This system effectively modified epigenetic marks on histone residues and reduced levels of target gene mRNA in cultured neurons.

These two technical tours-deforce provide neuroscientists with the ability to control naturally occurring processes with high temporal and spatial precision.

Leonie Welberg

ORIGINAL RESEARCH PAPERS Lin, J. Y. et al.

Optogenetic inhibition of synaptic release with chromophore-assisted light inactivation (CALI). Neuron 79, 241-253 (2013)|Konermann, S. et al. Optical control of mammalian endogenous transcription and epigenetic states. Nature http://dx.doi.org/10.1038/nature12466 (2013) 